\title{
PERSPECTIVAS E NOVAS TECNOLOGIAS PARA PRODUÇÃO DE LAGOSTAS PALINURÍDEOS
}

\author{
Perspectives and new technologies for production of palinurid spiny lobster
}

\begin{abstract}
Marco Antonio Igarashi
Professor associado do D epartamento deEngenharia dePesca da UniversidadeFederal do Cearáàdisposição da Secretaria Especial de Aquicultura e Pesca da Presidência da República, unidade descentralizada de Londrina, PR - Brasil, e-mail: igarashi@ ufc.br
\end{abstract}

\section{Resumo}

A presente revisão descreve a importância do cultivo de lagostas e demonstra a situação atual desta produção no mundo. Em países como Vietnam, as lagostas são produzidas em sistemas de engorda comercial. Entretanto, no Brasil o cultivo de juvenis de lagostas palinurídeos ainda é pouco conhecido devido aos seus longos estágios de desenvolvimento. O s resultados demonstram que a engorda de lagostas é considerado um importante agronegócio em termos de geração de emprego e lucros em moeda estrangeira em alguns países. Juvenis cultivados em cativeiro encontram um mercado valoroso. D essa forma, a indústria do cultivo de lagostas pode promover estabilidade socioeconômica das áreas costeiras, além de compensar a exploração da pesca tradicional. Ressalta-se que a indústria do cultivo de lagostas deve se unir a um adequado manejo e conservação dos recursos ambientais para sobreviver e prosperar.

Palavras-chave: Lagosta; Engorda; Produção.

\begin{abstract}
The present review describes the importance of spiny lobster culture and shows the status of this production in the world. In countries such as Vietnam, spiny lobsters are produced in commercial growout systems. However, juvenile culture in Brazil is still poorly understood, because juveniles posses the problems for culture of having long juvenile stage development. The results show that spiny lobster growout is already an important agribusiness in terms of job employment, foreign exchange earning, in some countries. A valuable market exists for juvenile spiny lobster cultured in captivity. In this forms, the industry can promote socio-economic stability of the coastal areas where the populations live, besides that the spiny lobster culture can replace fully exploited traditional fisheries as market demand suppliers. The industry must unite an adequate management and conservation of environmental resources to survive and thrive.
\end{abstract}

Keywords: Spiny lobster; Grow-out; Production. 


\section{INTRODUÇÃO}

As lagostas palinurídeos estão entre os crustáceos mais valiosos do mundo por serem classificados como alimento altamente valorizado. A captura mundial foi estimada em 77.000 toneladas por ano, com desembarque no valor aproximadamente de US\$ 500 milhões (PHILLIPS; KITTAKA, 2000; FRISCH, 2007).

O Brasil destaca-se como terceiro maior produtor mundial de lagostas com a captura de três espécies Panulirus argus, $\mathrm{P}$. laevicauda e P. echinatus, que representam uma entrada de divisas a ordem de 50 a 60 milhões de dólares (G Ó ES, 2006). No entanto, problemas relacionados ao alto consumo, à alta cotação no mercado internacional, à sobrepesca e à pesca predatória estão causando a diminuição das exportações desse recurso pesqueiro. Assim, uma das formas para aumentar a produção desse crustáceo é a partir da aquicultura.

0 maior problema para o desenvolvimento de técnicas em aquicultura de espécies de lagostas encontra-se na manutenção com êxito dos estágios larvais (filosoma). Essa fase possui duração longa, de 150 a 300 dias, (MIKAMI; KUBALA, 2004) e não há cultivo de larvas a nível comercial. Portanto a aquicultura sustentável de lagostas espinhosas necessita ser baseada na produção consistente de juvenis em laboratório (RITAR; THOMAS; BEECH, 2002).

Nesse contexto, entre os palinurídeos que foram cultivados até 0 assentamento, encontramse espécies como Jasus lalandii (KITTAKA, 1988), Sagmariasus (anteriormente Jasus) verreauxi (KITTAKA et al., 1997), Palinurus elephas (KITTAKA; IKEGAMI, 1988), P. japonicus (KITTAKA; KIMURA, 1989; YAMAKAWA et al., 1989), e P. longipes bispinosus (como P. longipes) (MATSUDA; YAMAKAWA, 2000).

Os requerimentos tecnológicos para larvicultura de lagostas espinhosas ( $\mathrm{J}$. edwardsii, P. japonicus e P. ornatus) estão correntemente sob investigação (MATSUD A; YAMAKAWA, 1997; MATSUDA; TAKENOUCHI; YAMAKAWA, 2003; BOURNE et al., 2004; SMITH et al., 2003, 2004; SMITH; RITAR, 2006). No entanto, apesar dos avanços na pesquisa, ainda é necessário desenvolver novas técnicas de larvicultura para alcançar o objetivo que é a produção comercial (SMITH; LYAL; RITAR, 2007).

As lagostas tropical (P. ornatus) e a vermelha (Panulirus argus) são as espécies mais indicadas para a aquicultura por possuírem o desenvolvimento larval mais curto (4 a 8 meses) (ACOSTA; MATTHEWS; BUTLER, 1997; CRUZ et al., 2001) e rápido crescimento pós-larval (WILLIAMS, 2007).

As lagostas adultas têm sido cultivadas em gaiolas na Nova Z elândia, Austrália, Índia, Brasil e Japão (BO OTH; KITTAKA, 2000; BARNEVELD, 2001; JAMES; MARIAN, 2003; BRYARS; GED D ES, 2005). A tualmente, a indústria da lagosta tropical P. ornatus em muitas partes do sudeste da Ásia e notavelmente no Vietnam, onde a abundância de pós-puerulus (juvenil) tem possibilitado 0 desenvolvimento da engorda de juvenis com produção de mais de 2.000 toneladas, com valor de US $\$ 70$ a 75 milhões em 2001/ 2002 (THUY; NGOC, 2004).

Recentemente a produção tem sido estimada em mais de 3.000 toneladas com 0 valor de exportação aproximado de US\$ 90 milhões (WILLIAM, 2007), porém pesquisas sobre a engorda de lagostas em gaiolas no mar permanecem escassas (JEFFS; JAMES, 2001, SIMO N; JAME S, 2007).

Tendo em vista os fatos anteriormente expostos, esta revisão foi realizada com o objetivo de somar informações sobre a situação do cultivo de lagostas, considerando os entraves tecnológicos e 0 ascendente potencial da atividade como fonte gerado ra de empregos e renda para o desenvolvimento sustentável.

\section{PRODUÇÃO}

Economicamente, a lagosta Panulirus argus e P. laevicauda são as espécies que sustentam a pesca comercial no Brasil, sendo a região Nordeste a principal produtora desse crustáceo . A produção majoritária cabe geralmente ao Ceará, a qual em 2005 atingiu 2.970 toneladas (Figura 1). 
Nos últimos anos, a exploração dos estoques lagosteiros localizados no Nordeste brasileiro aumentou de maneira significativa, refletindo o otimismo gerado pelas altas margens de lucro e, sobretudo, pela demanda cada vez maior do mercado internacional que absorve cerca de $90 \%$ da produção total do país (GÓ ES, 2006).

Há mercado para todas as lagostas capturadas (PHILLIPS; KITTAKA, 2000). A duração do suprimento (lagostas) e a competição entre os países estão mudando com a moderna tecnologia em operação.

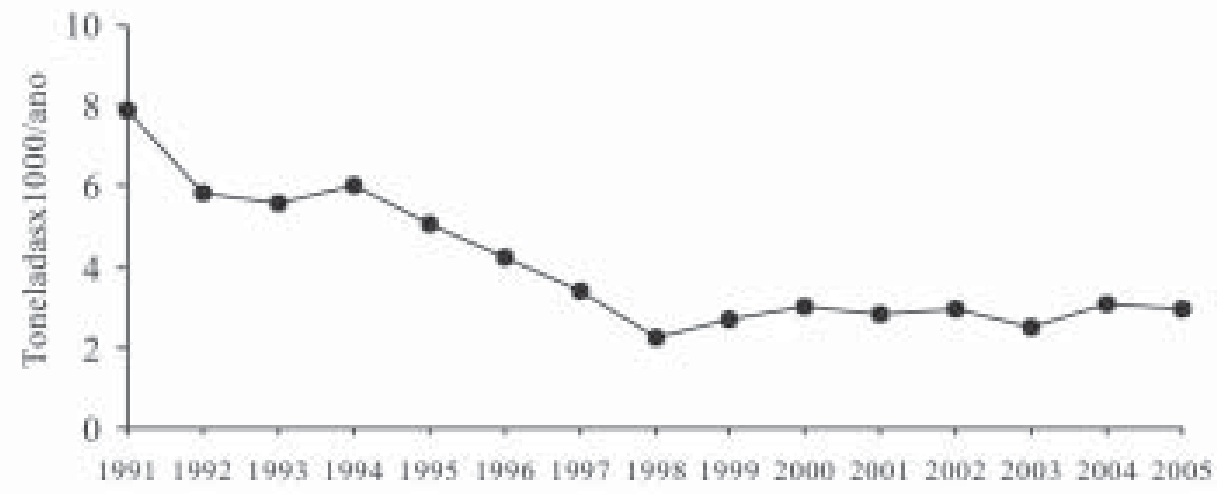

FIGURA 1 - Produção de lagosta desembarcada no Estado do Ceará durante 1991 a 2005

Figure 1 - L obster production seton shore in Ceará State during 1991 to 2005

Fonte: IBAMA/ ESTATIPESCA, 2006.

O valor comercial e econômico de cada espécie de lagosta pode variar em função de aspectos culinários, a exemplo de itens sensoriais ou organolépticos (sabor, textura, etc.) que lhe são atribuídos, em consequência da sua variação bromatológica.

Em geral, a lagosta brasileira, comercializada na forma de cauda geralmente resfriadas ou conservada em gelo, apresenta características de frescor aquém do produto similar australiano e neozelandês, comercializado na forma viva, refletindo na melhor cotação dessas últimas.

Entretanto, a lagosta explorada no Brasil pode apresentar problemas como a "barriga preta", ou seja, uma reação enzimática de escurecimento, por conta de manuseio indevido.

Esse agravante, que compromete 0 aspecto do produto em maior ou menor grau, pode ser contornado mediante a imersão das caudas numa solução de bissulfito de sódio. No entanto, essa solução, quando aplicada em concentrações acima de um determinado limiar e por tempo prolongado, pode deixar resíduo de substância perniciosa à saúde humana (IGARASHI; MAGALHÃ E S NETO , 1999).

Por conseguinte, convém orientar corretamente a aplicação do referido método de inibição, ou induzir o emprego da eutanásia a bordo, ou seja, imersão das lagostas vivas em água gelada (PHILLIPS; KITTAKA, 2000).

\section{REPRODUÇÃO}

O acasalamento de algumas espécies de lagostas ocorre após as mudas de fêmeas maduras. De acordo com Kittaka e Macdiarmid (1994) a muda provê a fêmea com cerdas ovígeras dos endopoditos dos pleópodos para a fixação dos ovos. Segundo os mesmos autores o comprimento das cerdas ovígeras aumenta a cada muda após a maturidade.

Em algumas espécies tropicais, de acordo com a maturação da gônada e a época do ano poderá ocorrer um segundo acasalamento (CREASER, 1950; MACFARLANE; MOORE, 1986; 
BRIO NES; LOZANO , 1992). Chittleborough (1976) observou que a muda e o acasalamento na fêmea poderia ser separado por 2 a 97 dias em P. cygnus. Em P. ornatus, o intervalo entre a muda e o acasalamento foi de 63 dias para o primeiro acasalamento e 25 dias para o segundo (MURUGAN et al., 2005).

0 macho de lagostas deposita o espermatóforo sobre o esterno da fêmea durante 0 acasalamento, e a fertilização dos óvulos ocorre quando a fêmea desova. O s ovos são retidos nas cerdas ovígeras dos endopoditos dos pleópodos.

0 período entre o acasalamento e a fertilização dos óvulos difere dependendo das espécies. Por exemplo, em P. japonicus isso pode levar menos do que uma hora, porém J. edwardsii pode carregar o espermatóforo por meses antes da fertilização (PHILLIPS; KITTAKA, 2000).

Após obter a maturidade sexual, as lagostas podem ter três desovas em 6 meses (CHITTLEBO RO UG H, 1976; VIJAYAKUMARAN et al., 2004a), sendo que quando alimentadas com dieta nutricionalmente balanceada em cativeiro, podem procriar 6 vezes ou mais (MURUGAN et al., 2005). No entanto, a média do número de larvas de P. ornatus (541.894 \pm 98.755) cultivadas (MURUGAN et al., 2005) é muito menor do que a média obtida de reprodutores selvagens (1.121.507 \pm 576.158 ) (VIJAYAKUMARAN et al. 2004b). Isso pode ocorrer devido ao fato dos reprodutores no primeiro ano da produção de ovos possuírem tamanho reduzido. Esse fato ocorre em P. polyphagus, que produz poucos ovos no primeiro ano de reprodução (KAGWADE, 1988ab). Outro fator que pode reduzir o número de larvas são as desovas repetidas dentro da mesma estação, como ocorre para várias espécies, tais como para P. argus (CREA SER, 1950), P. japonicus (INO, 1950), P. inflatus (BRIO NES; LOZANO, 1992), P. homarus (VIJAYAKUMARAN et al., 2004a) e P. ornatus (MACFARLANE; MOORE, 1986). Em P. homarus pode ocorrer equivalentemente uma segunda desova com um acasalamento (VIJAYAKUMARAN et al., 2004a).

No entanto, a lagosta apresenta potencialidade para o cultivo comercial a médio e a longo prazo por reproduzir e maturar em cativeiro, porém poucos trabalhos foram realizados com o objetivo de cultivo em larga escala no Brasil.

\section{LARVAS FILOSOMAS}

Os palinurídeos têm complexa história de vida (BOOTH, 1989). O cultivo completo de filosomas (Figura 2) das espécies de lagostas palinurídeos foi descrito por quatro instituições, três do Japão e uma da Nova Zelândia, porém o cultivo com êxito tem sido efetuado em escala limitada frequentemente usando sistema com tanque com capacidade para menos de 100 litros, e com menos de $1 \%$ de sobrevivência para o estágio juvenil.

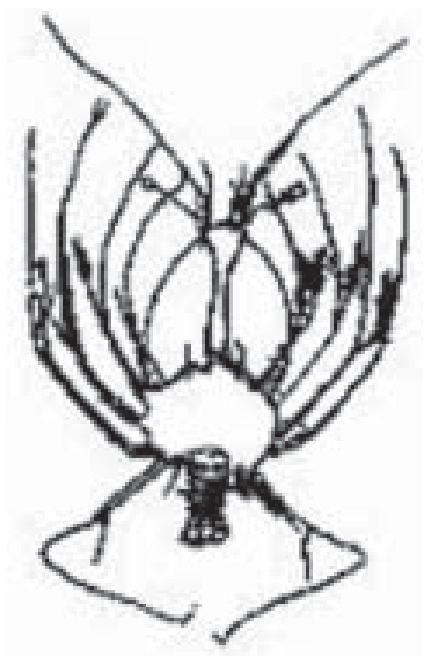

FIGURA 2 - Filosoma

Figure 2 - Filossoma 
Os ovos eclodem e desenvolvem as larvas filosomas, as quais, após uma série de mudas, metamorfoseiam-se para o estágio de puerulus (BO OTH, 1989).

As lagostas espinhosas têm a mais longa e complexa história larval de todos os crustáceos decápodas, envolvendo pelo menos 9 estágios morfológicos distintos e até 2 anos para metamorfose em puerulus (KITTAKA, 2000). 0 puerulus é o estágio intermediário entre a fase larval e juvenil.

0 puerulus morfologicamente é similar ao juvenil, mas é quase transparente e capaz de nadar para frente ou para trás usando os grandes pleópodos e curvando o abdômen (BO OTH, 1989).

A larva filosoma com acesso a um grande número de A rtemia aumenta seu consumo, o qual é comum em cultivo de crustáceo, e pode prover as reservas para o uso durante os estágios posteriores (INO UE, 1965; VIJAYAKUMARAN; RAD HAKRISHNAN, 1986; TO NG et al., 2000; THESSALO ULEGAKI et al., 1999).

Q uando a alimentação é insuficiente, as mudas podem se atrasar por vários dias (TO NG et al., 1997). A tualmente, a dieta padrão para cultivo de larvas filosoma é a A rtemia (SMITH; RITAR, 2006) devido ao fato de não ser fornecido alimento artificial.

Um dos maiores obstáculos no cultivo comercial de lagostas é obter sobrevivência satisfatória em todo o seu ciclo larval, fato pelo qual o cultivo de lagostas espinhosas não tem ainda se desenvolvido em larga escala, apesar de significantes avanços nas pesquisas. Progressos no cultivo até o estágio de puerulus têm sido realizados com várias espécies de lagostas, mas somente um pequeno número de puerulus tem sido produzido em laboratório (MURAKAMI; JINBO; HAMASAKI, 2007).

\section{ENGORDA}

O P. ornatus é a maior espécie tropical, chegando a mais de 6,5 kg (MURUGAN et al., 2005), atingindo $300 \mathrm{~g}$ no primeiro ano quando cultivada desde o estágio de puerulus (TAMN, 1980). Essa espécie engorda mais rápido no laboratório do que outras espécies de lagostas tropicais tais como $\mathrm{P}$. homarus, P. polyphagus, e P. versicolor (RADHAKRISHNAN; VIJAYAKUMARAN, 1990; VIJAYAKUMARAN; RADHAKRISHNAN, 1997).

Em função da rápida taxa de crescimento e atingir a maturidade sexual com o tamanho de $1 \mathrm{~kg}$, a P. ornatus está sendo considerada a espécie ideal para a aquicultura (MURUGAN et al., 2005).

Aproximadamente 15\% de P. ornatus exportado da Índia possui tamanho de 300 a $500 \mathrm{~g}$ (VIJAYAKUMARAN; RAD HAKRISHNAN, 1997). Lagostas com tamanho menor que 200 a $300 \mathrm{~g}$ podem ser capturadas na costa e serem engordadas por períodos curtos em tanques dentro de galpão ou em gaiolas no mar. Existe uma tecnologia básica onde a lagosta é alimentada com moluscos, crustáceos e peixes.

A pesar de apresentar um desenvolvimento mais rápido que as fêmeas, os machos perdem peso após começar o acasalamento (MURUGAN et al., 2005); já as fêmeas desviam as reservas energéticas do crescimento para a produção de ovos (BRYARS; GEDDES, 2005). Portanto a taxa de crescimento declina em fêmeas de J. edwardsii quando estas se tornam sexualmente maturas (PRE SCOTT et al., 1997). O bservações similares na redução de peso em fêmeas após iniciar a reprodução foram relatadas em cativeiro com reprodutores de P. homarus por Vijayakumaran et al. (2004a).

Assim, para aquicultura, machos e fêmeas devem ser cultivados separadamente após atingir a maturidade sexual para conseguir a taxa máxima de crescimento, prevenindo estes de se acasalar e procriar. Porém, este não deve ser o maior problema no cultivo de P. ornatus, desde que a maturidade seja atingida com tamanhos maiores do que 0 pico do tamanho comercial ( $<1 \mathrm{~kg})$ (MURUGAN et al., 2005). Machos apresentam substancialmente um maior ganho de peso do que as fêmeas (média de $8 \%$ para fêmeas e $17 \%$ para machos) (BRYARS; GEDDES, 2005).

No Vietnam, as lagostas são exclusivamente alimentadas com rejeito fresco da pesca (WILLIAM, 2007). No entanto, há vários estudos em que as lagostas foram alimentadas com dieta natural, especialmente mexilhão (JAMES; TO NG, 1997, 1998; LO RKIN et al., 1999; CREAR et al., 1999). Os mexilhões têm demonstrado resultados superiores em experimentos de engorda de lagostas 
quando comparados com alimentos peletizados. 0 trabalho experimental em laboratório encontrou que $0 \mathrm{~J}$. edwardsii adulto de $470 \mathrm{~g}$ consumiu somente 2 a $3 \mathrm{~g}$ de peso seco de alimento por dia, representando somente 0,4 a $0,6 \%$ do peso do corpo por dia (GEDDES et al., 2001).

Para crescerem as lagostas sofrem o fenômeno da muda, isto é, a troca do exoesqueleto. Prescott et al. (1997) encontrou dois picos da atividade da muda por ano em machos com comprimento da carapaça $<120 \mathrm{~mm}$, sendo um no verão e um segundo no inverno. Fêmeas pequenas com comprimento da carapaça $<90 \mathrm{~mm}$ podem mudar duas vezes ao ano. Fêmeas com comprimento da carapaça de até $110 \mathrm{~mm}$ mudam uma vez por ano. Fêmeas e machos com comprimento da carapaça $>120$ mm podem mudar uma vez por ano (BRYARS; GEDDES, 2005).

A maneira possível de melhorar o ganho de peso na muda inclui o aumento na frequência alimentar (ROBERTSO N et al., 1993), fornecimento da alimentação ao anoitecer (THO MAS; CARTER; CREAR, 2003) e diminuição da densidade no cultivo (JAMES et al., 2001).

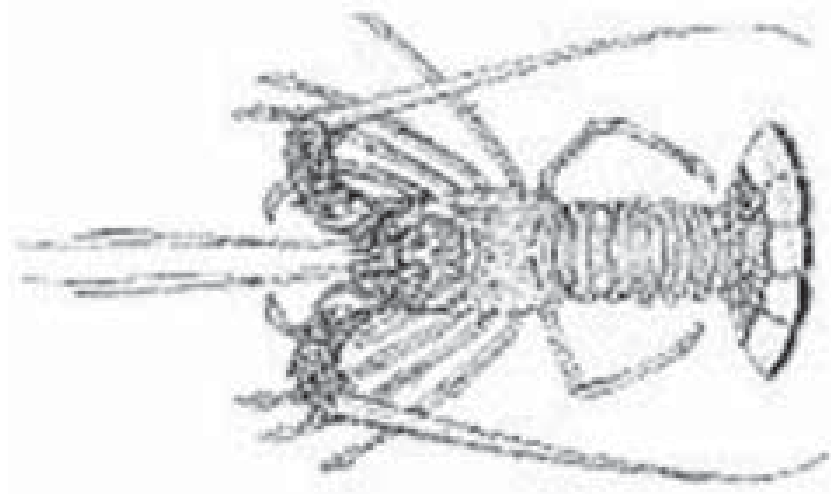

FIG URA 3 - Lagosta Panulirus argus

Figure 3 - L obster Panulirus argus

A variação na densidade da população, temperatura da água, assim como a taxa de predação e injúria afetam a taxa de crescimento da população local (BOOTH; KITTAKA, 2000).

Com a elaboração de uma metodologia de cultivo comercial, poder-se-á melhorar a qualidade de vida das comunidades litorâneas, alavancando oportunidades técnicas, a partir da produção de lagostas que satisfaça às necessidades dos mercados de exportação, propiciando, dessa forma, uma situação mais favorável para a economia brasileira.

\section{CONSIDERAÇÕES FINAIS}

A prioridade maior nas investigações está na produção de pueruli a partir da larvicultura comercial. Além disso, a formulação de dieta artificial e mais pesquisas sobre doenças são necessárias. A tualmente, a doença não é considerada o maior problema, pois não há indício de doenças em lagostas capturadas na natureza.

As pesquisas devem envolver desde a captura de pueruli e/ ou juvenis no ambiente, bem como seu posterior cultivo sob regime de confinamento. Essa possibilidade há de se considerar onde ocorrem juvenis de P. argus e de P. laevicauda em profusão no Brasil.

Para o desenvolvimento de tecnologia de cultivo de lagostas, o Brasil vem aprofundando e/ ou complementando os estudos até então realizados. Nesse sentido entende-se que os dados científicos disponíveis no país são suficientes para a implantação de um projeto-piloto de cultivo comercial de lagostas. 


\section{AGRADECIMENTOS}

Agradeço ao Professor Jiro Kittaka da Universidade de Ciência de Tokyo pelas informações fornecidas sobre o cultivo de lagostas.

\section{REFERÊNCIAS}

ACOSTA, C. A.; MATTHEWS, T. R.; BUTLER, M. J. Temporal patterns and transport processes in recruitment of spiny lobster (Panulirus argus) postlarvae to south Florida. Marine Biology, v. 129, n. 1, p. 79-85, 1997.

BARNEVELD, R. D evelopments in rock lobster enhancement and aquaculture III: third annual rock lobster enhancement and aquaculture subprogram workshop - Wellington. New Zealand: RLEAS Publication, 2001.

BOOTH, J. D. Occurrence of the puerulus stage of the rock lobster, Jasus edwardsii a the New Plymouth Power Station, New Zealand. New Zealand Journal of Marine and Freshwater Research, v. 23, n. 1, p. 43-50, 1989.

.; KITTAKA, J. Spiny lobster growout. In: PHILLIPS, B. F.; KITTAKA, J. (Ed.). Spiny lobsters: fisheries and culture. 2nd ed. Oxford: Fishing News Books, 2000. p. 556-585.

BRIO NES- FO URZAN, P.; LOZANO-ALVAREZ, E. Aspects of reproduction of Panulirus inflatus (Bouvier) and P. gracilis Streets (D ecapoda: Palinuridae) from the pacific coast of Mexico. Journal of Crustacean Biology, v. 12, n. 1, p. 41-50, 1992.

BRYARS, S. R.; GEDDES, M. C. Effects of diet on the growth, survival, and condition of seacaged adult southern rock lobster, Jasus edwardsii. N ew Zealand Journal of Marine and Freshwater Research, v. 39, n. 2, p. 251-262, 2005.

CHITTLEBOROUGH, R. G. Breeding of Panulirus longipes cygnus George under natural and controlled conditions. Australian Journal of Marine and Freshwater Research, v. 27, n. 3, p. 499-516, 1976.

CREAR, B. J.; THOMAS, C. W.; HART, P. R. An evaluation of the suitability of artificial prawn feeds as diets for the southern rock lobster, Jasus edwardsii'. In: THE ANNUAL INTERNATIO NAL CONFERENCE AND EXPOSITION OF THE WORLD AQUACULTURE SOCIETY, 1., 1999. Sydney. Proceedings... Australia: World Aquaculture Society, NSW, 1999. p. 183.

CREASER, E. P. Repetition of egg laying and number of eggs of the Bermuda spiny lobster. Proceedings of the Gulf Caribbian Fisheries Institute, v. 2, n. 1, p. 30-31, 1950.

CRUZ, R. et al. Variability in recruitment of multiple life stages of the Caribbean spiny lobster, Panulirus argus, in the gulf of batabano, Cuba. Marine Freshwater Research, v. 52, n. 8, p. 12631270, 2001.

FRISCH, A. J. Growth and reproduction of the painted spiny lobster (Panulirus versioolor) on the Great Barrier. Reef Fisheries Research, v. 85, n. 1, p. 61-67, 2007.

GEDDES, M. C. et al. Rock lobster enhancement and Aquaculture Subprogram project 5: determination of the optimum environmental and system requirements for juvenile and adult rock lobster holding. D eakin: Fisheries Research and D evelopment Corporation, 2001. 
GÓES, C. A. Análise da dispersão de larvas de lagostas no Atlântico Tropical a partir de correntes geostróficas superficiais derivadas por satélites. 2006. 93 f. Dissertação (Mestrado em Sensoriamento Remoto) - INPE, São José dos Campos, 2006. Disponível em: <http:/ / mtcm17.sid.inpe.br/col/ sid.inpe.br/ MTC-m13@80/ 2006/07.10.14.12 / doc/ publicacao. pdf>. Acesso em: 07 jul. 2007.

INSTITUTO BRASILEIRO DO MEIO AMBIENTE E DOSRECURSOSNATURAISRENOVÁVEIS. IBAMA/ ESTATIPESCA - IBAMA. Estatística da Pesca do IBAMA. 2006. Em Disquete.

IGARASHI, M. A.; MAGALHÃES NETO, E. O. O ciclo de desenvolvimento e a situacão atual do cultivo de lagostas no Brasil e no mundo. Revista Econômica do Nordeste, v. 30, n. 1, p. 94-99, 1999.

INO, S. O bservation on the spawning cycle of Ise-ebi (Panulirus japonicus v. Siebold). Nippon Suisan Gakkaishi, v. 15, n. 5, p. 725-727, 1950.

INOUE, M. On the relation of amount of food taken to the density and size of food and water temperature in rearing the phyllosoma of the Japanese spiny lobster, Panulirus japonicus (V. Siebold).

Bulletin of the Japanese Society of Scientific Fisheries, v. 31, n. 6, p. 903-906, 1965.

JAMES, C. M.; MARIAN, P. Lobster fattening and fishery in India. Marine and Freshwater Research, v. 1, n. 3, p 9-11, 2003.

JAMES, P. J:; TO NG, L. J. D ifferences in growth and moult frequency among post-pueruli of Jasus edwardsii fed fresh, aged or frozen mussels. Marine and Freshwater Research, v. 48, n. 8, p. 931-934, 1997.

Feeding technique, critical size and size preference of Jasus edwardsii fed cultured and wild mussè-Is. Marine and Freshwater Research, v. 49, n. 1, p. 151-156, 1998.

; PAEWAI, M. The effect of stocking density and shelter on growth and mortality of early juvenile Jasus edwardsii. Marine and Freshwater Research, v. 52, n. 8, p. 1413-1417, 2001.

JEFFS, A. G.; JAMES, P. Sea-cage culture of the spiny lobster Jasus edwardsii in New Zealand. Marine and Freshwater Research, v. 52, n. 8, p. 1419-1424, 2001.

KAGWADE, P. V. Reproduction in the spiny lobster, Panulirus polyphagus (Herbst). Journal of the Maine Biological Association of India, v. 30, n. 1-2, p. 37-46, 1988a.

Fecundity in the spiny lobster, Panulirus polyphagus (Herbst). Journal of the Maine Biōogical Association of India, v. 30, n. 1-2, p. 114-120, 1988b.

KITTAKA, J. Culture of the palinurid Jasus lalandii from egg stage to puerulus. Bulletin of the Japanese Society of Scientific Fisheries, v. 54, n. 1, p. 87-93, 1988.

. Culture of larval spiny lobsters. In: PHILLIPS, F.; KITTAKA, J. (Ed.). Spiny lobster: fisheries and culture. London: Fishing News Books, Blackwell Scientific Publications, 2000. p. 508-532.

; IKEG AMI, E. Culture of palinurid Palinurus elephas from egg stage to puerulus. Bulletin of the Japanese Society of Scientific Fisheries, v. 54, n. 7, p. 1149-1154, 1988.

; KIMURA, K. Culture of the Japanese spiny lobster Panulirus japonicus from egg to juvenile stage. Bulletin of the Japanese Society of Scientific Fisheries, v. 55, n. 6, p. 963-970, 1989.

.; MACD IARMID, A. B. Breeding. In: PHILLIPS, B. F.; COBB, J. S.; KITTAKA, J. Spiny lobster management. London: Fishing News, 1994. p. 384-401.

; O NO, K.; BO OTH, J. D. Complete development of the green rock lobster, Jasus verreauxi from egg to juvenile. Bulletin of Marine Science, v. 61, n. 1, p. 57-71, 1997.

LORKIN, M. et al. Sea-based live holding of the southern rock lobster, Jasus edwardsii: a pilot study on long term holding feeding. SARDI Research Report Series, v. 1, n. 46, p. 22, 1999. 
MACFARLANE, J. W.; MOORE, R. Reproduction of the ornate rock lobster, Panulirus ornatus (Fabricius), in Papua New Guinea. Australian Journal of Marine and Freshwater Research, V. 37, n. 1, p. 55-65, 1986.

MAT SUDA, H.; TAKENOUCHI, T.; YAMAKAWA, T. Diel timing of molting and metamorphosis of Panulirus japonicus phyllosoma larvae under laboratory conditions. Fisheries Science, v. 69, n. 1, p. 124-130, 2003.

MATSUDA, H.; YAMAKAWA, T. Effects of temperature on growth of the Japanese spiny lobster, Panulirus japonicus (V. Siebold) phyllosomas under laboratory conditions. Marine and Freshwater Research, v. 48, n. 7, p. 791-796, 1997.

The complete development development and morphological change of larval P. longipes (Decapoda, Palinuridae) under laboratory conditions. Fisheries Science, v. 66, n. 2, p. 278-293, 2000.

MIKAMI, S.; KUBALLA, A. Overview of lobster aquaculture research. In: THE SECOND HATCHERY FEED S AND TECHNOLOGY WORKSHOP, 1., 2004. Sydney. Anais... Sydney: Novatel Century Sydney, 2004. p. 127-130.

MURAKAMI, K.; JINBO, T.; HAMASAKI, K. A spects of the technology of phyllosoma rearing and metamorphosis from phyllosoma to puerulus in the Japanese spiny lobster Panulirus japonicus reared in the laboratory. Bulletin of Fisheries Reseach Agency, v. 2, n. 20, p. 59-67, 2007.

MURUGAN, T. S. et al. G rowth, repetitive breeding, and aquaculture potential of the spiny lobster, Panulirus ornatus. N ew Zealand Journal of Manine and Freshwater Research, v. 39, n. 3, p. 311-316, 2005.

PHILLIPS, B. F.; KITTAKA, J. Spiny lobster: fisheries and culture. Oxford: Blackwell Scietific Publications Fishing News Books, 2000.

PRE SCO TT, J. et al. Population dynamics of southern rock lobster in South Australian waters. Camberra: Fisheries Research and D evelopment Corporation, 1997.

RADHAKRISHNAN, E. V.; VIJAYAKUMARAN, M. An assessment of potential of spiny lobster culture in India. Bulletin of Central Manine Fisheries Research Institute, v. 44, part 2, p. 416-427, 1990.

RITAR A. J.; THOMAS C. W.; BEECH, A. J. Feeding A rtemia and shellfish to phyllosoma larvae of southern rock lobster (Jasus edwardsii). Aquaculture, v. 212, n. 1-4, p. 179-190, 2002.

ROBERTSO N, L.; LAWRENCE, A. L.; CASTILLE, F. L. Effect of feeding frequency and feeding time on growth of Penaeus vannamei (Boone). Aquaculture and Fisheries Management, v. 24, n. 1, p. 1-6, 1993.

SIMO N, C. J.; JAMES, P. J. The effect of different holding systems and diets on the performance of spiny lobster juveniles, Jasus edwardsii . Aquaculture, v. 266, n. 1-4, p. 166-178, 2007.

SMITH, G. G.; LYALL, L.; RITAR, A. J. The effect of predator/ prey density and water dynamics on feed intake and growth in spiny lobster larvae (Jasus edwardsii). Aquaculture, v. 263, n. 1-4, p. 122-129, 2007.

SMITH, G. G. et al. Effects of starvation and feeding on the fatty acid profiles of Stage I phyllosoma of the spiny lobster, Jasus edwardsii. Aquaculture, v. 34, n. 5, p. 419-426, 2003.

SMITH, G.; BROWN, M.; RITAR, A. Feeding juvenile A rtemia enriched with ascorbic acid improves larval survival in the spiny lobster Jasus edwardsii. Aquaculture, v. 10, n. 1, p. 105-112, 2004.

SMITH, G. G.; RITAR, A. J. The influence of animal density and water turbulence on growth and survival of cultured spiny lobster (Jasus edwardsii) larvae. Aquaculture, v. 258, n. 1-4, p. 404-411, 2006.

TAMM, G. R. Spiny lobster culture: an alternative to natural stock assessment. American Fisheries Society Bulletin, v. 5, n. 4, p. 59-62, 1980. 
THESSALOU-LEGAKI, M.; PEPPA, A.; ZACHARAKI, M. Facultative lecithotrophy during larval development of the burrowing shrimp C allianassa tyrrhena (D ecapoda: Callianassidae). Marine Biology, v. 133, n. 1, p. 635-642, 1999.

THOMAS, C. W.; CARTER, C. G.; CREAR, B. J. Feed availability and its relationship to survival, growth, dominance and the agonistic behaviour of the southern rock lobster, Jasus edwardsii in captivity. Aquaculture, v. 215, n. 1-4, p. 45-65, 2003.

THUY, N. T. B.; NG OC, N. B. Current status and exploitation of wild spiny lobsters in Vietnamese waters. In: WILLIAMS, K. C. (Ed.). Spiny Lobster Ecology and Exploitation in the South China Sea Region. Camberra: Australian Centre for International Agricultural Research, 2004. p. 13-16.

TONG, L. J. et al. Pickering TD effect of brine-shrimp numbers on growth and survival of earlystage phyllosomal larvae of the rock lobster Jasus edwardsii. Marine and Freshwater Research, v. 48, n. 8, p. 935-940, 1997.

TONG, L. J. et al. Effect of temperature and feeding rate on the growth and survival of early and mid -stage phyllosomas of the spiny lobster Jasus edwardsii. Marine and Freshwater Research, v. 51, n. 2, p. 235-241, 2000.

VIJAYAK UMARAN, M. et al. Captive breeding of the spiny lobster Panulirus homarus (Linnaeus, 1758). In: INTERNATIONAL CONFERENCE AND WORKSHOP ON BIOLOGY AND MANAGEMENT OF LOBSTERS, 7., 2004a, Tasmania. Proceedings... Tasmania: Hobart, 2004a. p. 99.

VIJAYAKUMARAN, M. et al. Fecundity and viability of eggs in wild breeders of the spiny lobsters, Panulirus homarus (Linnaeus, 1758), Panulirus versioolor (Latrielle, 1804) and Panulirus ornatus (Fabricius, 1798). In: INTERNATIO NAL CONFERENCE AND WORKSHOP ON BIOLOGY AND MANAGEMENT OF LOBSTERS, 7., 2004b, Tasmania. Proceedings... Tasmania, Hobart, 2004b. p. 147.

VIJAYAKUMARAN, M.; RADHAKRISHNAN, E. V. Spiny lobster fattening and live transport. In: OF THE WORKSHOP, NATIONAL AQUACULTURE WEEK, 1., 1997, Chennai. Proceedings... Chenai: Aquaculture Foundation of India, 1997. p. 97-103,

Effects of food density on feeding and moulting of phyllosomas larvae of the spiny Tōsste Panulirus homarus (Linnaeus). In: SY MPO SIUM OF COASTAL AQUACULTURE, 4., 1986. India. Proceedings... India: Marine Biological Association of India. 1986. p. 1281-1285.

WILLIAMS, K. C. Nutritional requirements and feeds development for post-larval spiny lobster: a review. Aquaculture, v. 263, n. 1-4, p. 1-14, 2007.

YAMAKAWA, T. et al. Complete larval rearing of the Japanese spiny lobster Panulirus japonicus. Nippon Suisan Gakkaishi, v. 55, n. 5, p. 745, 1989.

Recebido: 19/05/ 2008

Received: 05/ 19/2008

Aprovado: 09/ 09/ 2008

A pproved: 09/ 09/2008 